\title{
New insights about the evaluation of human sperm quality: the aromatase example
}

\author{
I. Galeraud-Denis ${ }^{1,2}$, C. Travert ${ }^{2}$, C. de Vienne' ${ }^{2}$ L. Said ${ }^{2,3}$, A. Saad ${ }^{3}$ and S. Carreau ${ }^{2}$ \\ ${ }^{1}$ Unité de Biologie de la Reproduction - CHU Caen - Avenue Georges Clémenceau - 14033 Caen cedex \\ France \\ 2UPRES EA 2608-USC INRA, Université de Caen - Esplanade de la Paix - 14032 Caen cedex - France \\ ${ }^{3}$ Laboratoire Cytogénétique et Biologie de la Reproduction, Hôpital Universitaire F Hached, \\ 4000-Sousse-Tunisie
}

\begin{abstract}
Male contribution to the couple's infertility is at first evaluated by the routine examination of semen parameters upon optical microscopy providing valuable information for a rational initial diagnosis and for a clinical management of infertility. But the different forms of infertility defined according to the WHO criteria especially teratozoospermia are not always related to the chromatin structure or to the fertilization capacity. New investigations at the molecular level (transcript and protein) could be developed in order to understand the nature of sperm malformation responsible of human infertility and thus to evaluate the sperm quality. The profile analysis of spermatozoal transcripts could be considered as a fingerprint of the past spermatogenic events. The selection of representative transcripts of normal spermatozoa remains complex because a differential expression (increased, decreased or not modified levels) of specific transcripts has been revealed between immotile and motile sperm fractions issued from normozoospermic donors. Microarrays tests or real-time quantitative PCR could be helpful for the identification of factors involved in the male infertility. Differences in the expression of specific transcripts have been reported between normal and abnormal semen samples. With the aromatase example, we have noted a negative strong correlation between the amount of transcript and the percentage of abnormal forms especially in presence of head defects. Immunocytochemical procedures using fluorescent probes associated with either confocal microscopy or flow cytometry can be also helpful to proceed with further investigations about the localization of proteins in the compartmentalized spermatozoa or the acrosome reaction. The dual location of aromatase both in the equatorial segment, the mid-piece and the tail could explain the double role of this enzyme in acrosome reaction and motility.
\end{abstract}

Key words: male infertility, routine semen analysis, spermatozoa, RNAs, confocal microscopy

\section{Introduction}

The spermatozoon is a highly differentiated cell characterized by a compartmentalized morphological structure and programmed to carry the paternal haploid genome throughout the male and female genital tracts in order to deliver it into the oocyte.

Spermiogenesis and epididymal maturation are essential steps for the acquisition of the final spermatozoal structure organization related to the protection and the transport of the genetic material throughout the genital tracts or the fertilization capacity on contact with the oocyte. But in the uterus and/or the fallopian

Correspondence: I. Galeraud-Denis, CHU Caen - Unité de Biologie de la Reproduction, Avenue Georges Clémenceau, 14033 - Caen cedex, France; e-mail: denis-i@chu-caen.fr tubes, spermatozoa must perform the pre-requisite steps of capacitation and acrosome reaction.

The semen analysis is the initial routine male investigation in couples with an history of infertility. Assessment of reproductive fitness has relied upon microscopic evaluation of sperm parameters including total sperm count, sperm concentration, percent of motile sperm and percent of normal sperm morphology thus providing valuable information about a rational initial diagnosis (azoospermia) or a clinical management of the infertility. However the definition of different forms of infertility (normozoospermia, asthenozoospermia, teratozoospermia or oligoasthenozoospermia) widely depends on the threshold values of sperm parameters based mainly on the World Health Organization standard [42]. This classification is essential for the choice of IVF treatment 
but cannot be considered as a predictive tool for the evaluation of sperm quality and for the IVF outcomes.

\section{Relation between semen parameters and sperm functions}

The relevance of the utility of semen parameters for assessing male infertility is still discussed. Normozoospermia is not always synonymous with fertilization. Despite of the use of sophisticated methods such as DNA fragmentation, acrosome reaction tests improving the prediction of fertilization capacity, fertilization failure cannot be excluded after conventional IVF. In patients with bordeline semen, the decision to choose either IVF or ICSI is critical because the risk of fertilization failure in conventional IVF is also difficult to predict [40]. Another problem results from the potential differences in sperm characteristics when semen analyses are performed before and during of oocyte retrieval. Globally $25-50 \%$ of cycles are rescued by ICSI when sibling oocytes are treated with conventional IVF and ICSI $[36,40]$.

Teratozoospermia is defined as the decrease of percent of normal sperm morphology but the threshold value differs according to the WHO criteria $(<30 \%)$ [42] or to Gusick et al. $(<11 \%)$ [17]. Abnormal sperm morphology is classified as defects in the head, mid-piece or tail of the sperm with the helpful of either the modified David [6] or Kruger [24] classifications the last being more correlated with the likelihood of fertilization in IVF. Sperm morphology has been reported to be the most important sperm parameter for predicting fertilization success in IVF [17]. Chocat et al have identified using a ROC curve a cutoff value of $17 \%$ of normal forms that can predict the absence of fertilization in $72 \%$ of patients when conventional IVF is performed [1].

Discordant studies of the effect of severe teratozoospermia on the fertilization capacity in IVF cycles have been reported. A better fertilization rate could be observed in ICSI compared with IVF [22,35]. But recently, Keegan et al [20] have noted that isolated teratozoospermia using Kruger/Tyneberg strict criteria does not affect in vitro fertilization outcome and is not an indication for intracytoplasmic sperm injection. But in their study, we can note the high proportion of isolated teratozoospermia in the population studied $(55 \%$ with lower than $5 \%$ of normal forms). Severe teratozoospermia does not affect blastocyst formation, clinical pregnancy and live birth rates in ICSI cycles [10]. These two recent reports suggest that sperm morphology especially assessed by Kruger's strict criteria seem to have a weak progostic value in assisted reproductive technology cycles.

Teratozoospermia is a common term gathering spermatozoa with different morphological phenotypes and thus aetiologies. Three groups of teratozoospermia have been globally studied. The first one concerns single structural defects resulting from a genetic origin and involving all the ejaculated spermatozoa such as either acrosome agenesis or globozoospermia or primary cilia dyskinesia [9]. The second one resulting from meiotic abnormalities gathers "enlarged head spermatozoa" with multiple tail and abnormal acrosome. The last group representing the most common cases is characterized by a polymorphic teratozoospermia where spermatozoa possess more than one morphological defect.

It is now apparent that some male factor infertility phenotypes associated with increased DNA fragmentation and/or chromosome aneuploidies may have effects on early embryogenesis [32]. However a specific association between the degree of teratozoospermia and sperm chromosome abnormalities has not been found using fluorescence in situ hybridization even if teratozoospermia like asthenozoospermia or oligozoospermia has revealed a slight increase in the frequency of chromosome abnormalities [39]. In men with normal karyotype, aneuploidy is present in almost all the "enlarged head teratozoospermia" but its frequency is slighty increased in the "polymorphic teratozoospermia" [28].

The potential adverse effects of sperm DNA damage on fertilization and embryo developpement could be explored by the sperm chromatin structure assay parameters (SCSA), DNA fragmentation (DFI) and high DNA stainability (HDS). Despite of a small but significant relation between sperm DNA integrity and pregnancy rate in IVF and ICSI cycles, this marker is not sufficient to provide a clinical indication for routine [2]. IVF and ICSI fertilization rates, embryo quality and pregnancy rate are not influenced by the amount of either DFI or HDS which might be related to spontaneous abortion rates [27].

Besides the classical sperm parameters (concentration, motility and morphology) and the SCSA parameters, new investigations at the molecular level (transcript or protein) could be necessary for the understanding of the sperm structure organization in relation to its functions (fertilization and implication in early embryonic development). The determination of sperm transcripts profile or the measurement of subcellular sperm functions using fluorescence signals could improve our knowledge about sperm quality and thus to assess male infertility.

\section{Human sperm transcripts as candidate markers of infertility}

Spermiogenesis is associated with profound architectural changes such as acrosome formation, DNA packaging, loss of cytoplasm, tail and mitochondrial sheath organization which are essential steps for the transformation of spermatids into functional spermatozoa. 
A wide range of transcripts synthetized either throughout the early spermatogenesis or during spermiogenesis has been found to be stored in translationally repressed ribonucleoproteins in round spermatids before their delayed activation for translation during spermiogenesis. During the last two decades numerous works have revealed an extended pattern of mRNA molecules using RT-PCR and/or in situ hybridization techniques [12-30], but also the presence of small RNAs (microRNAs, siRNAs) in the human spermatozoa [for review 5].

The use of either a step-by-step analysis associated with macroarrays, RT-PCR and in situ hybridization [4], or microarrays assay $[33,37]$ or serial analysis of gene expression (SAGE) [43] has confirmed the diversity and the complexity of mRNA present in ejaculated and uncapacitated spermatozoa. These spermatozoal transcripts are mainly located within the head region probably as a structural component of the nuclear matrix in association with histone bound DNA and the nuclear membrane $[5,25]$ suggesting their putative role either in the first divisions of the embryonic development [34] and/or in the epigenetic repackaging of sperm chromatin [31].

Until recently the male genome was thought to be on a transcriptionnally dormant state in spermatozoa [14]. Thus transcripts present in ejaculated spermatozoa could be considered as untranslated stored remnants reflecting the past spermatogenic and spermiogenetic events [29]. The first difficulty to analyse the spermatozoal mRNAs concerns the preparation of spermatozoal RNA devoid of any other somatic cell types or immature germ cells since individually they contain a greater amount of RNA than a single human spermatozoon carrying just 10-20 fg [23]. Thus, spermatozoa from native semen are selected using double swim-up or density gradient centrifugation followed by a hypotonic treatment [33] and/or the identification of somatic cell markers (CD 45 and E-cadherin) [26].

The analyses of the transcripts pattern in fertile and infertile patients could be used as a clinical tool [33]. In the future, the challenge is to define by microarrays technology the correct profile of transcripts present in normal spermatozoa and to justify the choice of transcripts as candidate markers of proven fertility taking account of individual variations of mRNAs distribution (absence and/or constant presence, large range in transcript signal). Using semi-quantitative PCR, we have compared the levels of spermatozoal transcripts coding for molecules involved in nuclear condensation (Prm1 and Prm2), capacitation (eNOS, nNOS), motility and sperm survival (P450arom and c-myc) in immotile and motile sperm fractions issued from normospermic donors. mRNA levels were either increased (eNOS, nNOS, Prm-1) or identical (c-myc, protamine 2) or decreased (aromatase) in low motile fraction compared to high motile fraction [26]. Variations of transcripts profiles are observed in the two fractions although the percentages of viable or apoptotic spermatozoa have been found unchanged. These data are concordant with those of Wang et al. [41] revealing a differential expression of two genes (TPX-1 and LDHC) in normal and motility impaired semen samples.

Differences in specific transcript levels in spermatozoa have been also observed between normal and abnormal sperm samples. Sperm mRNAs encoding for protamines have been largely studied and could be considered as candidate markers for the discrimination between fertile and infertile men [38] or between normozoospermic and asthenozoospermic men [21]. The quantitative assessment of Hils1, transition proteins 1 and 2 mRNAs by real-time quantitative PCR has shown a reduction of these transcripts in asthenozoospermic compared to normozoospermic men [19]. Only protamines (but not HILS1) and fertilin beta mRNA levels are well correlated to the embryo quality [7].

Regarding the expression of highly specific transcripts, very scarce studies have demonstrated the differential expression of specific transcripts which do not interfere with the chromatin reorganization. Microarrays tests have shown a differential mRNA distribution between normozoospermic and teratozoospermic patients [37]. The expression of VASA mRNA and protein were significantly reduced in oligozoospermic semen samples [16]. In our laboratory using real-time quantitative PCR, we have also analyzed 57 samples (18 normozoospermia N, 16 asthenozoospermia A, 11 asthenoteratozoospermia $\mathrm{AT}$ ). A significant reduction of the ratio $\mathrm{A} / \mathrm{G}$ is observed in the groups T (52\%) and AT (67\%). In the group AT, most of the samples have no detectable aromatase transcripts. A tight and negative correlation has been reported between the levels of aromatase mRNA and the spermatozoal morphology, especially in the case of microcephaly or acrosome malformations. By contrast, no differences have been observed when asthenozoospermic have been compared to normozoospermic semen samples. But asthenozoospermic patients could be divided in two subgroups characterized either by a low aromatase mRNA amount ( $\sim 50 \%$ decrease) or by an elevated level in relation to a higher $\mathrm{pH}$, a lower viability and a decrease of seminal alpha-glucosidase suggesting that asthenozoospermia is a form of infertility covering a large range of aetiologies [13]. The measure of aromatase transcripts levels could be helpful for a better characterization of asthenozoospermia.

\section{Use of fluorescent labels with confocal microscopy or flow cytometry}

Sperm functions are related to the compartmentalised structure of the spermatozoa: head implicated 
in fertilization steps (capacitation, acrosome reaction and/or fusion), tail responsible for the transport of chromosomal materiel and mid-piece involved in energy metabolism. Even in the sperm head, four regions (apical region, pre-equatorial region, equatorial segment and post-acrosomal region) have been isolated interacting specifically with the oocyte: the apical region playing a role in the binding of the oocyte zona pellucida although the pre-equatorial zone interfering mainly with acrosome reaction and the equatorial segment with fusion [11]. Immunocytochemical procedures using fluorescent signals associated with either confocal microscopy or flow cytometry could be also helpful to proceed with further investigations about the specific location of proteins or enzymes $[3,15]$. Using confocal microscopy, we have co-localised two sites of aromatase: annular aromatase in the equatorial region of the head and intense staining in the mid-piece/tail. Differences of protein distribution can be observed in normal and abnormal spermatozoa [8]. The aromatase protein distribution can be modified in uncapacitated spermatozoa of teratozoospermic patients or after induction of the capacitation. The proportion of intact acrosomes could also be evaluated in uncapacitated spermatozoa via CD46 ([15], Galeraud-Denis unpublished results). The acrosomal index established according the criteria of acrosomal Albert's classification seems to be overestimated by optical compared to confocal microscopy and to be decreased in T patients [13]. Using time-lapse fluorescence microscopy and CD 46 a marker of inner acrosomal membrane, Harper et al have shown that the acrosome reaction is a highly adapted form of exocytosis [18].

To conclude, analysing mRNAs profile in man could be helpful either as a diagnostic tool to assess male infertility since they reflect spermatogenesis gene expression and/or a prognosis value for fertilization and embryo development since these RNAs are delivered to oocyte. The analysis of different acrosome membrane proteins by confocal microscopy could represent a new form of investigation for the evaluation of the acrosome reaction.

Because of the potential implication of aromatase and estrogen receptors in sperm cells during capacitation and/or acrosome reaction, the aromatase transcripts and protein could be also used as markers of sperm quality using respectively real time PCR and confocal microscopy.

\section{References}

[ 1] Chocat A., Creveuil C., Galeraud-Denis I., Herlicoviez D., Herlicoviez M., Sauvalle A. Valeur prédictive des parametres spermatiques non automatisés et des parametres cinétiques automatisés sur le taux de clivage en fécondation in vitro. Gynecol Obstet Fertil. 2001;29:301-307.

[2] Collins JA, Barnhart KT, Schlegel PN. Do sperm integrity tests predict pregnancy with in vitro fertilization? Fertil Steril. 2008;89:823-831.
[3] Cummerson JA, Flanagan BF, Spiller DG, Johnson PM. The complement regulatory proteins CD55 (decay accelerating factor) and CD 59 are expressed on the inner acrosomal membrane of human spermatozoa as well as CD46 (membrane cofactor protein). Immunology. 2006;118:333-342.

[4] Dadoune JP, Pawlak A, Alfonsi MF, Siffroi JP. Identification of transcripts by microarrays, RT-PCR and in situ hybridization in human ejaculate spermatozoa. Mol Hum Reprod. 2005; 11:133-140.

[5] Dadoune JP. Spermatozoal RNAs : What about their functions? Microsc Res Techn 2009 accepted for publication

[6] David G, Bisson JP, Czyglik F et al. Anomalies morphologiques du spermatozoide humain. - Propositions pour un systeme de classification. J Gynecol Obstet Biol Reprod. 1975; suppl. 1:17-36.

[ 7] Depa-Martinow M, Kempisty B, Lianeri M, Jagodzinski PP, Jedrzejczak P. Association between fertilin beta, protamines 1 and 2 and spermatid-specific linker histone H1-like protein mRNA levels, fertilization ability of human spermatozoa, and quality of preimplantation embryos. Folia Histochem Cytobiol. 2007;45:S79-S85.

[ 8] de Vries KJ, Ziedmer T, Sims PJ, Gadella BM. Caspase-independent exposure of aminophospholipids and tyrosine phosphorylation in bicarbonate responsive human sperm cells. Biol Reprod. 2003;68:2122-2134.

[ 9] Francavilla S, Cordeschi G, Pellicicione F, Bocchio M, Francavilla F. Isolated teratozoospermia: a cause of male sterility in the era of ICSI? Front Biosci. 2007;12:69-88.

[10] French DB, Sabanegh ES Jr, Goldfarb J, Desai N. Does severe teratozoospermia affect blastocyst formation, live birth rate and other outcome parameters in ICSI cycles. Fertil Steril. 2009 (accepted for publication)

[11] Gadella BM, Tsai PS, Boerke A, Brewis IA. Sperm head membrane reorganisation during capacitation. Int J Dev Biol. 2008;52:473-480.

[12] Galeraud-Denis I, Lambard S, Carreau S. Relationship between chromatin organization, mRNAs profile and human gamete quality. Asian J Androl. 2007;9:587-592.

[13] Galeraud-Denis I, de Vienne CM, Said L, Chocat A, Carreau S. Differential expression of aromatase in human spermatozoa from normozoospermic and teratozoospermic patients. Int $J$ Androl. 2008;31(S1):1

[14] Grunewald S, Paasch U, GlanderHJ, Anderegg U. Mature human spermatozoa do not transcribe novel RNA. Andrologia. 2005;37:69-71.

[15] Grunewald S, Rasch M, Reinhardt M, Baumann T, Paasch U, Glander HJ. Stability of fluorochrome based assays to measure subcellular sperm functions. Asian $J$ Androl. 2008; 10:455-459.

[16] Guo X, Gui YT, Tang AF, Lu LH, Gao X, Cai ZM. Differential expression of VASA gene in ejaculated spermatozoa from normozoospermic men and patients with oligozoospermia. Asian J Androl. 2007;9:339-344.

[17] Gusick DS, Overstreet JW, Factor-Litvak P, Brazil CK, Nakajima STM, Coutifaris C et al. Sperm morphology, motility and concentration in fertile and infertile men. New Engl $J$ Med. 2001;345:1388-1393.

[18] Harper CV, Cummerson JA, While MRH, Publicover SJ, Johnson PM. Dynamic resolution of acrosomal exocytosis in human sperm. J Cell Sci. 2008;121:2130-2135

[19] Jedrzejczak P, Kempisty B, Bryja A, Mostowska M, DepaMartynow M, Pawelczyk L, Jagodzinski PP. Quantitative assessment of transition proteins 1,2 spermatid linker histone H1-like protein transcripts in spermatozoa from normozoospermic and asthenozoospermic men. Arch Androl. 2007;53:199-2005.

[20] Keegan BR, Barton S, Sanchez X, Berkeley AS, Krey LC, Grifo J. Isolated teratozoospermia does not affect in vitro fer- 
tilization outcome and is not an indication for intracytoplasmic injection. Fertil Steril. 2007;88:1583-1588.

[21] Kempisty B, Depa-Martinow M, Lianeri M, Jedrzejczak P, Darul-Wasowicz A, Jagodzinski PP. Evaluation of protamines 1 and 2 transcript contents in spermatozoa from asthenozoospermic men. Folia Histochem Cytobiol. 2007;45:S109113.

[22] Kihaile PE, Misumi J, Hirotsuru K, Kumasako Y, Kisanga RE, Utsunomiya T. Comparison of sibling oocytes outcomes after intracytoplasmic and in vitro fertilization in severe teratozoospermia in the first cycle. Int J Androl. 2003;26:57-62.

[23] Krawetz SA. Paternal contribution: new insights and future challenges. Nat Rev Genet. 2005;6:633-642.

[24] Kruger TF, Menkveld R, Stander FS, Lombard CJ, Van der Merwe JP, van Zyl JA et al. Sperm morphologic features as a prognostic factor for in in vitro fertilization. Fertil Steril. 1986;46:118-1123.

[25] Lalancette C, Miller D, Li Y, Krawetz SA. Paternal contributions: new functional insights for spermatozoal RNA. J Cell Biochem. 2008;104:1570-1579.

[26] Lambard S, Galeraud-Denis I, Martin G, Levy R, Chocat A, Carreau S. Analysis and significance of mRNA in human ejaculated sperm from normozoospermic donors : relationship to sperm motility and capacitation. Mol Hum Reprod. 2004; 10:535-541.

[27] Lin MH, Kuo-Kuang lee R, Li SH, Lu CH, Sun FJ, Hwu YM. Sperm chromatin structure assay parameters are not related to fertilization rates, embryo quality, and pregnancy in in-vitro fertilization and intracytoplasmic sperm injection, but might be related to spontaneous abortion rates. Fertil Steril. 2008;90:352-359.

[28] Machev N, Gosset P, Viville S. Chromosome abnormalities in sperm from infertile men with normal somatic karyotypes: teratozoospermia. Cytogenet Genome Res. 2005;111:352357.

[29] Miller D, Briggs D, Snowden H, Hamlington J, Rollison S, Lilford R, Krawetz SA. A complex population of RNAs exists in human ejaculate spermatozoa: implications for understanding molecular aspects of spermiogenesis. Gene. 1999;237: 385-392.

[30] Miller D, Ostermeir GC, Krawetz SA. The controversy, potential and roles of spermatozoal RNA. Trends Mol Med. 2005; 11:156-163.

[31] Miller D and Ostermeier GC. Towards a better understanding of RNA carriage by ejaculate spermatozoa. Hum Reprod Update. 2006;12:757-767.
[32] Nanassy L, Carrell DT. Paternal effects on early embryogenesis. J Exp Clin Assist Reprod. 2008;5:1-9.

[33] Ostermeier GC, Dix DJ, Miller D, Khatri P, Krawetz SA. Spermatozoal RNA profiles of normal fertile men. Lancet. 2002;360:772-777.

[34] Ostermeier GC, Dix DJ, Huntriss JD, Diamond MP, Krawetz SA. Reproductive biology : delivering spermatozoan RNA to the oocyte. Nature. 2004;429:154.

[35] Pisarska MD, Casson PR, Cisneros PL, Lamb DJ, Lipshultz LI, Buster JE et al. Fertilization after standard in vitro fertilization versus cytoplasmic sperm injection in subfertile males using sibling oocytes. Fertil Steril. 1999;71:627-632.

[36] Plachot M, Belaisch-Allart J, Mayenga JM, Chouraqui A, Tesquier L, Serkine AM. Outcome of conventional IVF and ICSI on sibling oocytes in mild male factor infertility. Hum Reprod. 2002;17:362-369.

[37] Platts AE, Dix DJ, Chemes HE, Thompson KE, Goodrich R, Rockett JC et al. Success and failure in human spermatogenesis as revealed by teratozoospermic RNAs. Hum Mol Genet. 2007; 16:763-773.

[38] Steger K, Wilhelm J, Konrad L, Stalf T, Greb R, Diemer T. Both protamine-1 to protamine- 2 mRNA ratio and $\mathrm{Bcl} 2$ mRNA content in testicular spermatids and ejaculated spermatozoa discriminate between fertile and infertile men. Hum Reprod. 2008;23:11-16.

[39] Sun F, Ko E, Martin RH. Is there a relationship between sperm chromosome abnormalities and sperm morphology? Reprod Biol Endocrinol. 2006;4:1-5.

[40] Van der Westerlaken L, Naaktgeboren N, Verburg H, Dieben $\mathrm{S}$, Helmerhorst FM. Conventional in vitro fertilization versus intracytoplasmic sperm injection in patients with borderline semen : a randomized study using sibling oocytes. Fertil Steril. 2006;85:395-400.

[41] Wang H, Zhou Z, Xu L, Li J, Xiao J, Xu ZY, ShAJ. A spermatogenesis-related gene expression profile in human spermatozoa and its potential clinical applications. $J$ Mol Med. 2004;82:317-324.

[42] World Health Organization. WHO laboratory manual for the examination of human semen and sperm-cervical mucus interaction. 4th ed. Cambridge, England :Cambridge University Press 1999.

[43] Zhao Y, Li Q, Yao C, Wang Z, Zhou Y, Wang Y et al. Characterization and quantification of mRNA transcripts in ejaculated spermatozoa of fertile men by serial analysis of gene expression. Hum Reprod. 2006;21:1583-1590. 